\title{
The equation of Euler's line yields a Tzitzeica surface
}

\author{
Alfonso F. Agnew, Alexandru Bobe, Wladimir G. Boskoff, \\ Laurenţiu Homentcovschi, and Bogdan D. Suceavă
}

Alfonso F. Agnew received his Ph.D. from Oregon State University in 1999. He is now an associate professor at California State University at Fullerton. His main fields of research include affine differential geometry, asymptotic analysis, twistor theory, geometric algebra and their applications in physics.

Alexandru Bobe received his Ph.D. in 2007. Presently, he is an assistant professor at the Ovidius University in Constanţa, Romania. His main fields of research include affine differential geometry, Riemannian geometry, computational algebra and geometry.

Wladimir G. Boskoff is a professor at the Faculty of Mathematics and Computer Sciences of Ovidius University in Constanţa, Romania. His main fields of research include foundations of geometry, metric geometry, and differential geometry. He was awarded the Gheorghe Tzitzeica Prize in Mathematics from the Romanian Academy of Sciences.

Laurentiu Homentcovschi is a senior lecturer at the Faculty of Mathematics and Computer Sciences of Ovidius University in Constanţa, Romania, since 1999. His main fields of research include geometry and computational geometry.

Bogdan D. Suceavă received his Ph.D. from Michigan State University in 2002. He is now an associate professor at California State University at Fullerton. His main fields of research include metric geometry, Riemannian and Kaehlerian geometry, and history of mathematics.

In dem nachfolgenden Beitrag wird ein Zusammenhang zwischen der Dreiecksgeometrie und der Differentialgeometrie von Flächen hergestellt. Genauer gesagt, geht es um einen Zusammenhang zwischen der Euler-Geraden eines Dreiecks $A B C$ und einer speziellen Quadrik im $\mathbb{R}^{3}$, der sogenannten Tzitzeica-Fläche. Bezeichnen $(x, y)$ die Koordinaten des variablen Punktes $A$, so zeigt sich, dass die Steigung der Euler-Geraden bei fixierten Ecken $B, C$ eine Tzitzeica-Fläche beschreibt. Durch das Studium der Niveaulinien dieser Fläche finden die Autoren einen alternativen Beweis für die Tatsache, dass der geometrische Ort der Ecken $A$ des Dreiecks $A B C$ mit einer Euler-Geraden, die konstante Steigung besitzt, eine Ellipse oder eine Hyperbel bildet. 


\section{Introduction}

The relative position of Euler's line with respect to the sides of a triangle has raised geometers' interest since the very first paper on this topic, Leonhard Euler's classical work [3]. To mention just a few recent references where this question has appeared, we remind the reader of problem A1 in the W.L. Putnam competition from 1997, which explored the case when Euler's line is parallel to one of the sides of a triangle. Problem 10980 in the American Mathematical Monthly, proposed by Ye and $\mathrm{Wu}$ [11] (see Corollary 1 below), works with the same configuration. In the Editorial Comment following the solution of the problem [11], the editors of the Monthly have pointed out other contributions to the history of this problem, especially Gossard's presentation at an AMS conference in 1915. A generalization given by Paul Yiu, dating from 1999, is mentioned in [4]. In the interesting paper [7], Rodriguez, Manuel, and Semião characterize the geometric locus of points $A$ in the plane for which the Euler line of $\triangle A B C$ has a prescribed slope $m$, when $B$ and $C$ are fixed.

In this paper we expand upon these matters by connecting the issue with ideas from affine surface geometry. In particular, we construct a Tzitzeica surface, or proper affine sphere, which we call the Euler surface associated with the equation of the slope of the Euler line. The geometry of the Euler line may then be analyzed in terms of the Tzitzeica surface (and vice versa). In Section 2, we review the background material on the slope of the Euler line and existing results. In Section 3, we review the concept of a Tzitzeica surface and affine invariance. In particular, we introduce the aforementioned Euler surface and detail its relevant geometrical properties. In the final section, we discuss the relationship between the geometry of the Euler surface and the geometry of the Euler line, both generally and by way of a detailed example. As a by-product, we obtain an example of a Tzitzeica curve on a Tzitzeica surface that is not asymptotic. This yields a nice counterexample to the converse of a classical result of affine geometry, which states that any asymptotic curve on a Tzitzeica surface is Tzitzeica.

\section{The relative position of the Euler line in a triangle}

In this section we present a few results unified by the following:

Lemma 1. Let $A, B, C$ be three arbitrary points in the Euclidean plane. Denote by $m_{E}$ the slope of Euler's line in $\triangle A B C$ and by $m_{1}, m_{2}, m_{3}$ the slopes of the lines $B C, A C$, and $A B$, respectively. Then

$$
m_{E}=-\frac{m_{1} m_{2}+m_{2} m_{3}+m_{3} m_{1}+3}{m_{1}+m_{2}+m_{3}+3 m_{1} m_{2} m_{3}}
$$

Proof. We assume for the beginning that $\triangle A B C$ is acute. Consider $\triangle A B C$ in Fig. 1 , where $H$ is the orthocenter, $O$ is the circumcenter, $F$ is the foot of the altitude from $A$ on the side $B C, S$ is the projection of the circumcenter onto the altitude from $A$, and $M$ is the midpoint of the side $B C$. Measuring the slope of the angle between $B C$ and Euler's line 


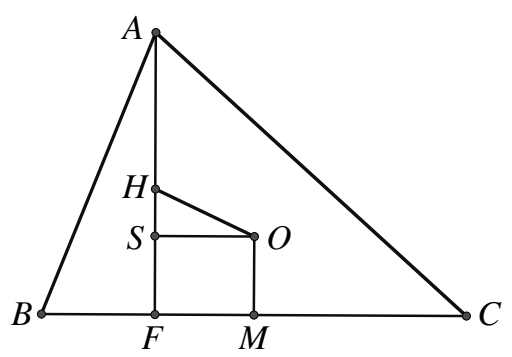

Fig. 1 The construction for Lemma 1

of $\triangle A B C$, we have (see Fig. 1)

$$
\begin{aligned}
\frac{m_{1}-m_{E}}{1+m_{1} m_{E}} & =\tan \angle(H O S)=\frac{H S}{M F}=\frac{A F-A H-O M}{B M-B F} \\
& =\frac{2 R \sin B \sin C-2 R \cos A-R \cos A}{R \sin A-2 R \sin C \cos B} \\
& =\frac{2 \sin B \sin C+3 \cos (B+C)}{\sin (B+C)-2 \sin C \cos B} \\
& =\frac{3 \cos B \cos C-\sin B \sin C}{\sin B \cos C-\sin C \cos B}=\frac{3-\tan B \tan C}{\tan B-\tan C} ;
\end{aligned}
$$

here, $R$ denotes the circumradius of $\triangle A B C$. Replacing in the last relation the following expressions

$$
\tan B=\frac{m_{3}-m_{1}}{1+m_{1} m_{3}}, \quad \tan C=\frac{m_{1}-m_{2}}{1+m_{1} m_{2}},
$$

we get the equality

$$
\frac{m_{1}-m_{E}}{1+m_{1} m_{E}}=\frac{3-\frac{m_{3}-m_{1}}{1+m_{1} m_{3}} \cdot \frac{m_{1}-m_{2}}{1+m_{1} m_{2}}}{\frac{m_{3}-m_{1}}{1+m_{1} m_{3}}-\frac{m_{1}-m_{2}}{1+m_{1} m_{2}}} .
$$

Cross-multiplying and collecting the like-terms, we obtain

$$
m_{1} m_{2}+m_{2} m_{3}+m_{3} m_{1}+m_{1} m_{E}+m_{2} m_{E}+m_{3} m_{E}+3 m_{1} m_{2} m_{3} m_{E}+3=0 .
$$

Solving for $m_{E}$ in this relation immediately yields the relation from the statement of our lemma.

A similar argument can be made if $\triangle A B C$ is obtuse.

Corollary 1. [11] Consider four distinct straight lines in the same plane with the property that no two of them are parallel, no three are concurrent, and no three form an equilateral triangle. Prove that, if one of the lines is parallel to the Euler line of the triangle formed by the other three, then each of the four given lines is parallel to the Euler line of the triangle formed by the other three. 
Proof. Denote by $m_{1}, m_{2}, m_{3}$, and $m_{4}$ the slopes of the four lines $d_{1}, d_{2}, d_{3}, d_{4}$, respectively. Suppose that Euler's line of the triangle formed by the lines $d_{1}, d_{2}, d_{3}$ is parallel to $d_{4}$ and has the slope $m_{E}$. Then $m_{E}=m_{4}$ and we get

$$
m_{1} m_{2}+m_{2} m_{3}+m_{3} m_{1}+m_{1} m_{4}+m_{2} m_{4}+m_{3} m_{4}+3 m_{1} m_{2} m_{3} m_{4}+3=0 .
$$

This relation is symmetric in any one of the slopes and the conclusion follows immediately.

Corollary 2. ([2], Problem 4 on p. 20) In the acute triangle ABC, Euler's line is parallel to $B C$ if and only if $\tan B \tan C=3$.

Proof. Choose a coordinate system so that the $x$-axis is parallel to $B C$. If we denote by $m_{1}$ the slope of the straight line $B C$, then $m_{1}=0$. Denoting $m_{2}, m_{3}, m_{E}$ the slopes of the straight lines $A C, A B$, and Euler's line $E$, respectively, we get from Lemma 1

$$
m_{E}=-\frac{m_{2} m_{3}+3}{m_{2}+m_{3}} .
$$

Thus, Euler's line $E$ of $\triangle A B C$ is parallel to $B C$ if and only if $m_{E}=0$, which is equivalent to $m_{2} m_{3}=-3$. Now we take into account that $m_{2}=-\tan C$ and $m_{3}=\tan B$ (or, depending on the position of $\triangle A B C$, we could have $m_{2}=\tan C$ and $m_{3}=-\tan B$ ). Consequently, $\tan B \tan C=3$.

Note. The geometric locus of the points $A$ in the plane (for $B$ and $C$ fixed) satisfying the property that Euler's line of $\triangle A B C$ is parallel to $B C$ is studied in [1]. The solution uses a direct trigonometric argument. We present in Section 4 the argument based on Lemma 1.

\section{Tzitzeica surfaces and the surface generated by the formula for Euler's line}

The study of Tzitzeica surfaces originates in $[8,9,10]$ and represents from the historical standpoint the beginning of affine differential geometry (see [6]). Some of the original references are still cited today (just to mention a recent example, see [5]).

Let $S$ be a surface given by the local parametrization $f: U=\stackrel{\circ}{U} \subset \mathbb{R}^{2} \rightarrow \mathbb{R}^{3}$ and denote by $K(p)$ its Gaussian curvature at $p \in f(U)$. We denote by $d(p)$ the distance between the origin in $\mathbb{R}^{3}$ and the tangent plane to the surface at $p$. A Tzitzeica surface is a surface $S$ for which there exists a constant $a \in \mathbb{R}$ such that for all points $p$ of the surface,

$$
K(p)=a \cdot d^{4}(p) .
$$

Tzitzeica proved that the class of surfaces satisfying the above condition is invariant under the action of the centro-affine group.

Next, consider a curve $c: I \subset \mathbb{R} \rightarrow \mathbb{R}^{3}$. Denote the torsion of $c$ by $\tau$. Then, the curve is called a Tzitzeica curve if it satisfies, for all $t \in I$, the relation $\tau(t)=a \cdot d(t)^{2}$, where $d(t)$ is the distance from the origin to its osculating plane at the point $c(t)$ and $a$ is a 


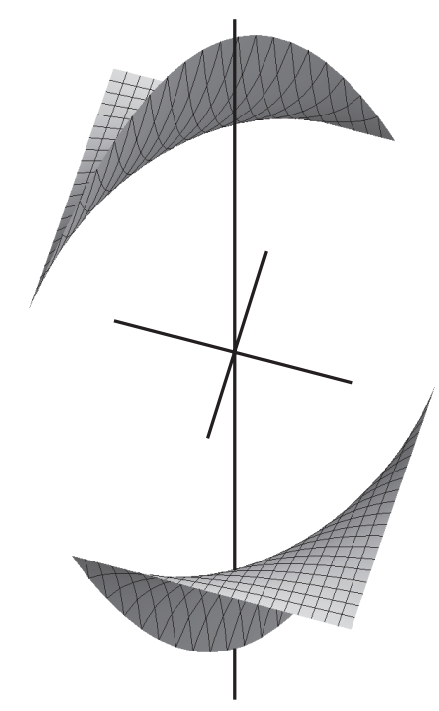

Fig. 2 A $\sqrt{3} \times \sqrt{3}$ square of the Euler surface, centered at the origin.

real constant. One of the classical results in affine differential geometry states that any asymptotic curve on a Tzitzeica surface is a Tzitzeica curve.

Lastly, consider an arbitrary triangle in the Euclidean plane. By making a convenient choice of coordinates axes, we can assume that one of the slopes (say, $m_{3}$ ) is zero. Then, as we have seen in the previous section, the slope of Euler's line becomes

$$
m_{E}=-\frac{3+m_{1} m_{2}}{m_{1}+m_{2}} .
$$

Define the surface $f: U \subset \mathbb{R}^{2} \rightarrow \mathbb{R}^{3}$ given by

$$
f(x, y)=\left(x, y,-\frac{3+x y}{x+y}\right),
$$

where $U$ denotes the complement of the line $x+y=0$ in $\mathbb{R}^{2}$. We present two views of the surface in Figures 2 and 3. In the first surface, we are looking at the image of a $\sqrt{3} \times \sqrt{3}$ square centered at the origin, while the second plot is zoomed out, covering the image of a $10 \times 10$ region. There is, of course, a planar vertical asymptote due to the singular line along $x=-y$, and graphical artifacts there should be ignored.

This surface has a large amount of symmetry, which is clear from the defining equation as well as the graph. First of all, $z$ is an odd function of $(x, y)$ in the sense that

$$
z(-x,-y)=-z(x, y)
$$

(in other words, $z$ changes sign under a $180^{\circ}$ rotation). Also, the surface is clearly symmetric with respect to the plane $x=y$. 


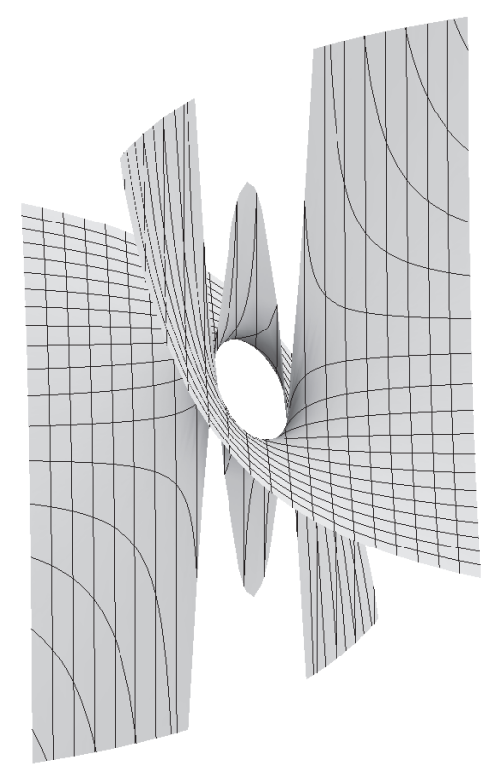

Fig. 3 A $10 \times 10$ square of the Euler surface from a different perspective, centered at the origin.

We now prove the following:

Theorem 1. The surface $f: U \stackrel{\circ}{U} \subset \mathbb{R}^{2} \rightarrow \mathbb{R}^{3}$ given by

$$
f(x, y)=\left(x, y,-\frac{3+x y}{x+y}\right)
$$

is a Tzitzeica surface.

Proof. By a standard computation, the Gaussian curvature is

$$
K=\frac{l \cdot n-m^{2}}{e \cdot g-f^{2}}=-\frac{3(x+y)^{4}}{\left(9+x^{4}+2 x^{3} y-3 y^{2}+2 x y^{3}+y^{4}+3 x^{2}\left(-1+y^{2}\right)\right)^{2}} ;
$$

here $l, m, n$ are the coefficients of the second fundamental form and $e, f, g$ are the coefficients of the first fundamental form of the surface defined by $f$. We also compute the support function $d$ (i.e. the distance from the origin to the tangent plane), resp. its fourth power:

$$
d^{4}=\frac{324(x+y)^{4}}{\left(9+x^{4}+2 x^{3} y-3 y^{2}+2 x y^{3}+y^{4}+3 x^{2}\left(-1+y^{2}\right)\right)^{2}} .
$$


We compute the necessary ratio and find it to be constant, whereby it is verified that the surface is Tzitzeica:

$$
\frac{K}{d^{4}}=-\frac{1}{108}
$$

Remark. The quadric $z=-\frac{3+x y}{x+y}$ is an open subset of a hyperboloid of one sheet, as one can see by reducing it to its canonical form (in new coordinates $x, y, z$ )

$$
-\frac{x^{2}}{3}+\frac{y^{2}}{6}+\frac{z^{2}}{6}=1
$$

In the last section we will discuss the details of its geometric relationship with the Euler line of a triangle in the plane.

\section{Geometric analysis}

So far we have pointed out that a specific quadric is a Tzitzeica surface. This result is known and would not be worthy of reporting by itself. However, the interesting aspect is the connection between affine surface geometry and triangle geometry in the plane. Using (2), we now discuss this connection. In particular, we are interested in clarifying the relationship between various loci of the vertex $A$ of an otherwise fixed triangle $\triangle A B C$ in the Euclidean plane and the geometry of the Euler surface. We will naturally obtain the results of [7] by considering the level curves of (3).

Suppose, without loss of generality, that in $\triangle A B C$, the points $B$ and $C$ are fixed at $(b, 0)$ and $(c, 0)$, where $b<c$. We will allow the coordinates $(x, y)$ of the vertex $A$ to vary. Then the slopes $m_{2}$ and $m_{3}$ (recall Lemma 1) are related to the coordinates of $A$ by

$$
m_{2}=\frac{y}{x-c}, \quad m_{3}=\frac{y}{x-b} .
$$

This yields a map $S: \mathbb{R}^{2} \rightarrow \mathbb{R}^{2},(x, y) \mapsto\left(m_{2}, m_{3}\right)$. The map $f \circ S$ results in a correspondence between the vertex $A$ and the slope of the Euler line given by

$$
m_{E}=\frac{-3(x-b)(x-c)-y^{2}}{y(2 x-b-c)},
$$

or

$$
3 x^{2}+2 m_{E} x y+y^{2}-\left(3 x+m_{E} y\right)(b+c)+3 b c=0 .
$$

A constant slope for the Euler line requires setting $m_{E}$ constant. For the Euler surface, this corresponds to setting $z$ constant, i.e., choosing a level curve of the surface. The level curves of (3) are of the form

$$
x y+(x+y) m_{E}+3=0,
$$

which is clearly a hyperbola for any fixed value of $m_{E}$. The effect on the loci defined by $A$ is determined by setting $m_{E}$ constant in equation (7). In this case (7) defines a quadratic curve in $\mathbb{R}^{2}$. The discriminant is given by

$$
\Delta=4\left(m_{E}^{2}-3\right) \text {. }
$$


Thus, we see that the locus of vertices $A$ which yield a fixed Euler line slope $m_{E} \in$ $(-\sqrt{3}, \sqrt{3})$ forms an ellipse, while for $m_{E} \in(-\infty,-\sqrt{3}) \cup(\sqrt{3}, \infty)$, the locus is a hyperbola. A short calculation shows that for the values $\pm \sqrt{3}$ we obtain double lines. These are the conclusions found in [7] using a different approach.

The other coordinate slices of the Euler surface yield hyperbolae. Upon, say, polynomial division, we find

$$
m_{E}=-m_{3}+\frac{m_{3}^{2}-3}{m_{2}+m_{3}}
$$

which shows explicitly that a hyperbola is obtained if we set $m_{3}$ to be constant. Due to the interchange symmetry between $m_{2}$ and $m_{3}$, the same will be true if we set $m_{2}$ to be constant. The effect of this restriction on the locus $A$ is straightforward by consideration of the equations (5). In particular, the hyperbolae map to lines: For values of the slope of the Euler line that vary with the slope $m_{2}$ (or $m_{3}$ ) only, that relationship must be inverse by (10), while the triangle vertex $A$ will trace out a line in the plane as a result of (5).

Being space curves lying in a plane, the curves arising from the above coordinate slices are all trivially Tzitzeica curves - curves with zero affine distance from the origin. These curves are not asymptotic curves of the surface, since in these cases the osculating plane does not overlap with the tangent plane to the surface. It is known that any asymptotic curve on a Tzitzeica surface is a Tzitzeica curve. Therefore, this class of curves provide counterexamples to the converse (Tzitzeica implies asymptotic).

On the other hand, viewed as curves in the 2-dimensional subspace of $\mathbb{R}^{3}$ corresponding to the coordinate slice, the hyperbolae are non-trivially Tzitzeica, in that the 2-d affine distance is constant but nonzero (affine circles). Thus, the level curves are all affine circles that map to loci $\{A\}$ that are also affine circles, while the loci corresponding to the other coordinate slices degenerate into lines.

Lastly, we provide here a more detailed analysis on a special case to make further reference to the literature. We begin by recalling a remark from [1]. Let $\triangle A B C$ be a triangle in the Euclidean plane with fixed vertices $B$ and $C$. The geometric locus of the points $A$ with the property that Euler's line of the triangle $A B C$ is parallel to $B C$ is the union of two open arcs of an ellipse from $B$ to $C$.

To see this, one can express $\tan B$ and $\tan C$ from the right triangles $A B F$ and $A F C$, respectively, to get (see Fig. 1):

$$
\frac{h_{a}}{B F} \cdot \frac{h_{a}}{F C}=3
$$

Hence $h_{a}^{2}=3 B F \cdot F C$. If we consider Cartesian coordinates so that $B=(0,0), C=$ $(c, 0)$, and $A=\left(x_{A}, y_{A}\right)$, then $F=\left(x_{A}, 0\right)$, and the previous equation can be written as:

$$
y_{A}^{2}=3 x_{A}\left(c-x_{A}\right) .
$$

One may visualize this piece of ellipse from $B$ to $C$, an arc between perpendiculars on $B C$ at $B$ and $C$, that we denote by $(E)$. For every point $A$ on this $\operatorname{arc}$, we have $\tan B \tan C=3$, and Euler's line for the triangle $A B C$ is parallel to $B C$ (see also [2, p. 20], the configuration mentioned above in Corollary 2). However, on this arc, there is a point where Euler's line is degenerate (and the corresponding position of $A$ is not on the geometric locus we discuss). 
When $A$ is at the vertex of this arc, by reasons of symmetry, the triangle $A B C$ has to be isosceles, therefore $\tan B \tan C=3$ yields $(\tan B)^{2}=3$, therefore $B=C=\frac{\pi}{3}$, so in this case the triangle has to be equilateral. However, in an equilateral triangle the Euler line is degenerate, since $O=H=G$, where $G$ is the center of gravity of $\triangle A B C$. This position does not belong to the geometric locus of the vertices $A$ with the property that Euler's line is parallel to the side $B C$. Therefore the geometric locus is an arc of ellipse from $B$ to $C$, with a gap of one point, corresponding to the position when $A B C$ is equilateral.

Let the vertex $A$ be on the ellipse described above. Then this position corresponds to a point in the fourth quadrant of the domain of the surface patch $f:(0, \infty) \times(-\infty, 0) \subset$ $\mathbb{R}^{2} \longrightarrow \mathbb{R}^{3}$ given by

$$
f\left(m_{1}, m_{2}\right)=\left(m_{1}, m_{2},-\frac{3+m_{1} m_{2}}{m_{1}+m_{2}}\right) .
$$

The domain is $(0, \infty) \times(-\infty, 0)$ since $m_{1}>0$ represents the slope of the side $A B$, and $m_{2}<0$ represents the slope of the side $B C$. More precisely, $\tan B=m_{1}$ and $\tan C=$ $-m_{2}$. Then, while $A$ is moving along the ellipse $(E)$ described above, $\left(m_{1}, m_{2}\right)$ traces the branch of the equilateral hyperbola $m_{1} m_{2}=-3$ that is mapped through $f$ on a curve on the surface $\operatorname{Im} f$. However, this curve represents the intersection of the surface $\operatorname{Im} f$ with the $m_{1} m_{2}$-plane in $\mathbb{R}^{3}$. It corresponds to the geometric locus of the points $A$ for which the Euler line is parallel to the side $B C$. More precisely, this curve can be written as

$$
c: \mathbb{R}-\{0\} \rightarrow \mathbb{R}^{3}, \quad c\left(m_{1}\right)=\left(m_{1},-\frac{3}{m_{1}}, 0\right)
$$

Of course, this curve is the intersection of the surface $\operatorname{Im} f$ with the $m_{1} m_{2}$-plane in $\mathbb{R}^{3}$.

\section{References}

[1] Boskoff, W.G.; Suceavă, B.D.: When Is Euler's Line Parallel to a Side of a Triangle? College Math. J. 35 (2004), 292-296.

[2] Coxeter, H.S.M.; Greitzer, S.L.: Geometry Revisited. Yale Univ. Press, 1967.

[3] Euler, L.: Solutio facilis problematum quorundam geometricorum difficillimorum. Novii comm. acad. sci. Petropolitanae 11 (1765), 103-123 (in Opera omnia, series 1, vol. 26, 139-157).

[4] Kimberling, C.: Web resource: Gossard Perspector, http: //faculty.evansville.edu/ck6/tcenters/recent/gosspersp.html

[5] Loftin, J.; Yau, S.-T.; Zaslow, E.: Affine manifolds, SYZ geometry and the "Y" vertex. J. Differential Geom. 71 (2005), 129-158.

[6] Nomizu, K.; Sasaki, T.: Affine differential geometry, Geometry of affine immersions. Cambridge Univ. Press, 1994.

[7] Rodriguez, J.; Manuel, P.; Semião, P.: A conic associated with Euler lines. Forum Geometricorum 6 (2006), 17-23.

[8] Țiţeica, G.: Sur une nouvelle classe de surfaces, I. Rend. Circ. Mat. Palermo 25 (1908), 180-187.

[9] Ţiţeica, G.: Sur une nouvelle classe de surfaces, II. Rend. Circ. Mat. Palermo 28 (1909), 210-216. 
[10] Ţiţeica, G.: Sur une nouvelle classe de surfaces. Comptes Rendus des Séances de l'Académie des Sciences Paris 150 (1910), 955-956.

[11] Ye, Z.H.; Wu, W.C.: Problem 10980. Amer. Math. Monthly (2002), 921; Solution (2004), 823-824.

\section{Alfonso F. Agnew}

Bogdan D. Suceavă

California State University Fullerton

Department of Mathematics

Fullerton, CA 92834-6850, USA

e-mail: aagnew@fullerton.edu

bsuceava@fullerton.edu

Alexandru Bobe

Wladimir G. Boskoff

Laurenţiu Homentcovschi

Department of Mathematics and Computer Science

University Ovidius

Constanţa, Romania

e-mail: alexb@univ-ovidius.ro

boskoff@univ-ovidius.ro

Ihomentcovschi@univ-ovidius.ro 